\title{
COVID-19 and the Incidence of Acute Myocardial Injury
}

\author{
Lars Mizera ${ }^{1}$ Oliver Borst ${ }^{1,2}$ \\ ${ }^{1}$ Department of Cardiology and Angiology, University of Tuebingen, \\ Tuebingen, Germany \\ ${ }^{2}$ DFG Heisenberg Group Thrombocardiology \\ Hamostaseologie 2021;41:356-364.
}

Address for correspondence Oliver Borst, MD, Department of Cardiology and Angiology, University Hospital Tuebingen, Otfried-Müller-Str. 10, 72076 Tuebingen, Germany

(e-mail: oliver.borst@med.uni-tuebingen.de).

Abstract
Keywords
- COVID-19
- myocardial infarction
- cardiac injury
- troponin

Cardiovascular manifestations are frequent in COVID-19 infection and are predictive of adverse outcomes. Elevated cardiac biomarkers are common findings in patients with cardiovascular comorbidities and severe COVID-19 infection. Troponin, inflammatory and thrombotic markers may also improve risk prediction in COVID-19. In our comprehensive review, we provide an overview of the incidence, potential mechanisms and outcome of acute cardiac injury in COVID-19. Thereby, we discuss coagulation abnormalities in sepsis and altered immune response as contributing factors favoring myocardial injury. We further highlight the role of endothelial damage in the pathophysiological concepts. Finally, observational studies addressing the incidence of myocardial infarction during COVID-19 pandemic are discussed.

\section{Introduction}

The current global pandemic of the coronavirus disease 2019 (COVID-19) presents a severe burden to healthcare services and economy. Up to this point, there are more than 231 million confirmed cases worldwide. The severe acute respiratory syndrome coronavirus 2 (SARS-CoV-2) affects predominantly the respiratory tract; however, extrapulmonary manifestations and multiple-organ involvement are also common. ${ }^{1}$ Among individuals, clinical presentation varies from asymptomatic subclinical infection, mild afebrile respiratory symptoms to pneumonia, and acute respiratory distress syndrome (ARDS). Cardiac biomarkers, including troponin and brain-natriuretic peptide, which reflect myocardial injury and dysfunction are commonly elevated in patients hospitalized with COVID-19., ${ }^{2,3}$ Recent studies by Majure et al. and Lombardi et al. showed that elevated troponin levels in COVID 19 patients are associated with increased mortality rates. ${ }^{4,5}$ Furthermore, there is increasing evidence that preexisting cardiovascular metabolic diseases may affect the prognosis. ${ }^{6}$ Known cardiovascular diseases such as hypertension, coronary heart disease, and congestive heart failure result in higher probability of infection with SARS-CoV-2 and a more severe course of COVID-19. In this review, we will focus on the incidence and pathophysiologic

received

May 12, 2021

accepted after revision

July 19, 2021 mechanism of cardiac injury and their implications for prognosis in COVID-19 patients.

\section{Methods}

The PubMed database was searched by the authors for literature published between February 2020 and April 2021 using the following search terms: "COVID-19" and "incidence myocardial infarction" or "cardiac injury." The title and abstract contents of the identified articles were reviewed and assessed for eligibility. Cross-references from relevant studies were added. The search was restricted to studies in English language.

\section{Results}

Our search yielded 1,499 results. After removing review articles, opinion letters, animal studies, duplications, and articles not relevant to cardiology, 212 studies were identified for full-text analysis. Of these, 20 studies were selected in our review for further analysis and their study characteristics are listed in - Table 1. Each study defined cardiac injury as elevation of serum troponin level above the 99th percentile upper reference limit according to the Fourth Universal Definition of Myocardial Infarction. ${ }^{7}$ For the definition of (c) 2021. Thieme. All rights reserved. Georg Thieme Verlag KG,

Rüdigerstraße 14,

70469 Stuttgart, Germany
DOI https://doi.org/ 10.1055/a-1554-6416. ISSN 0720-9355. 


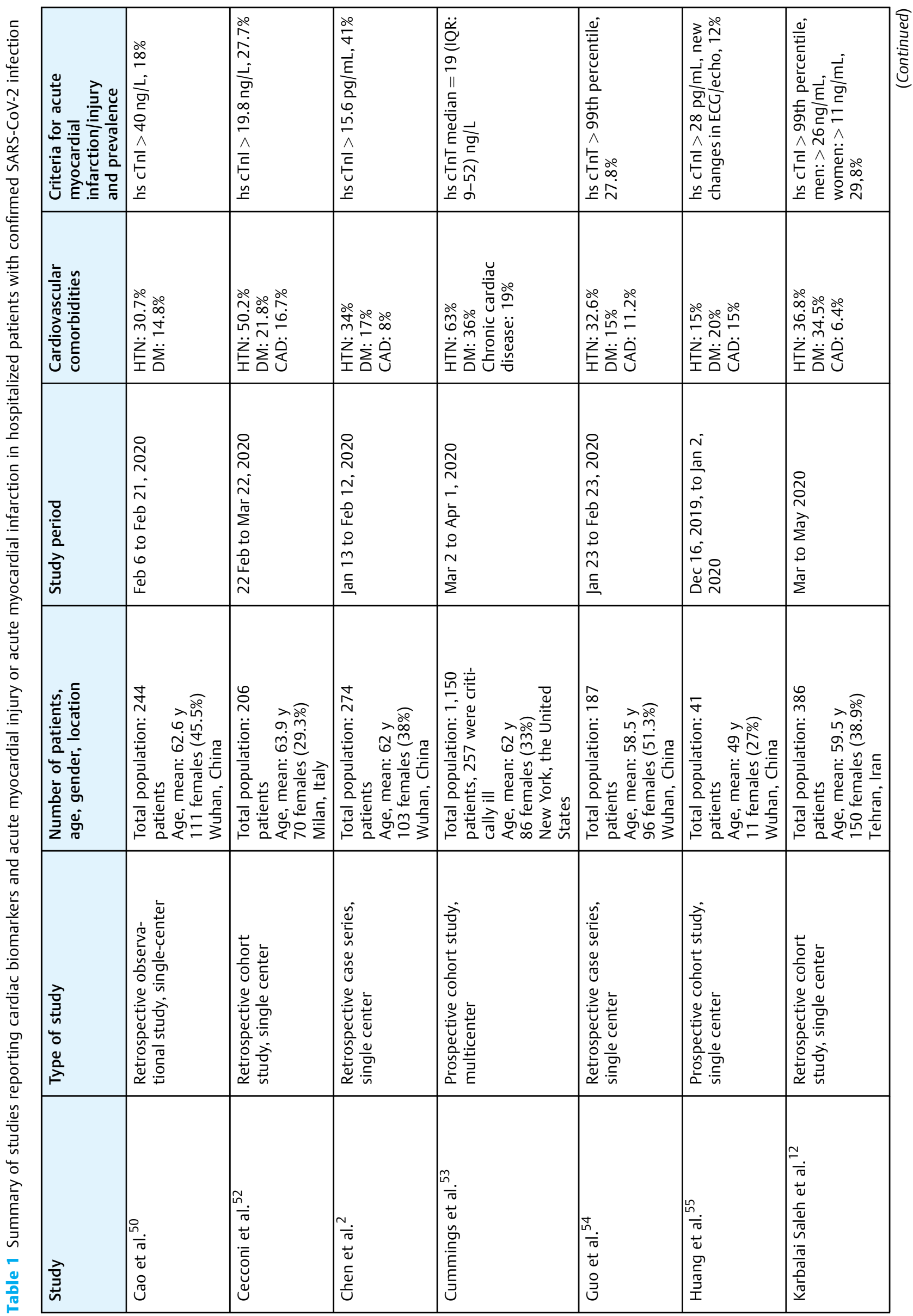




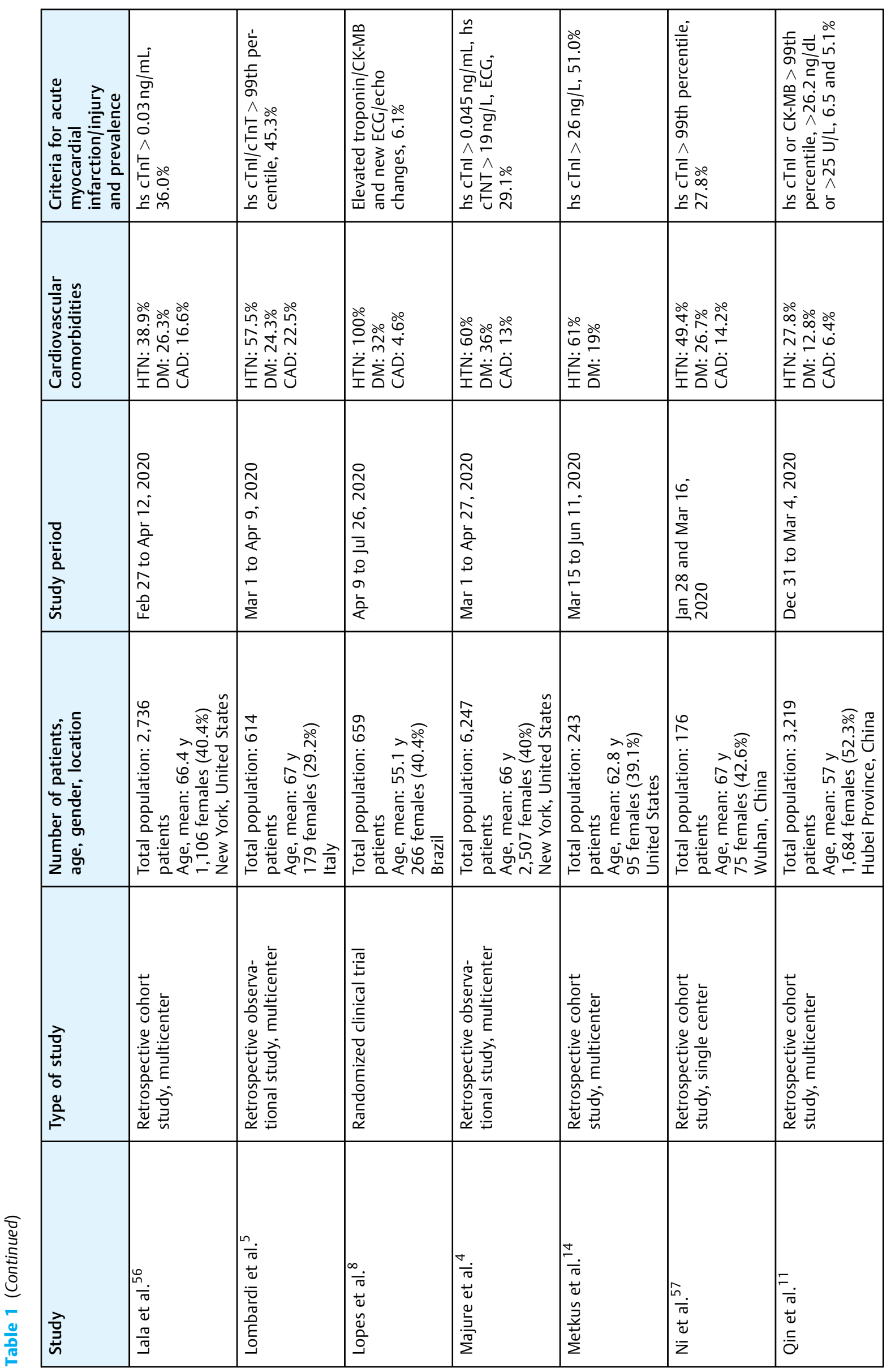




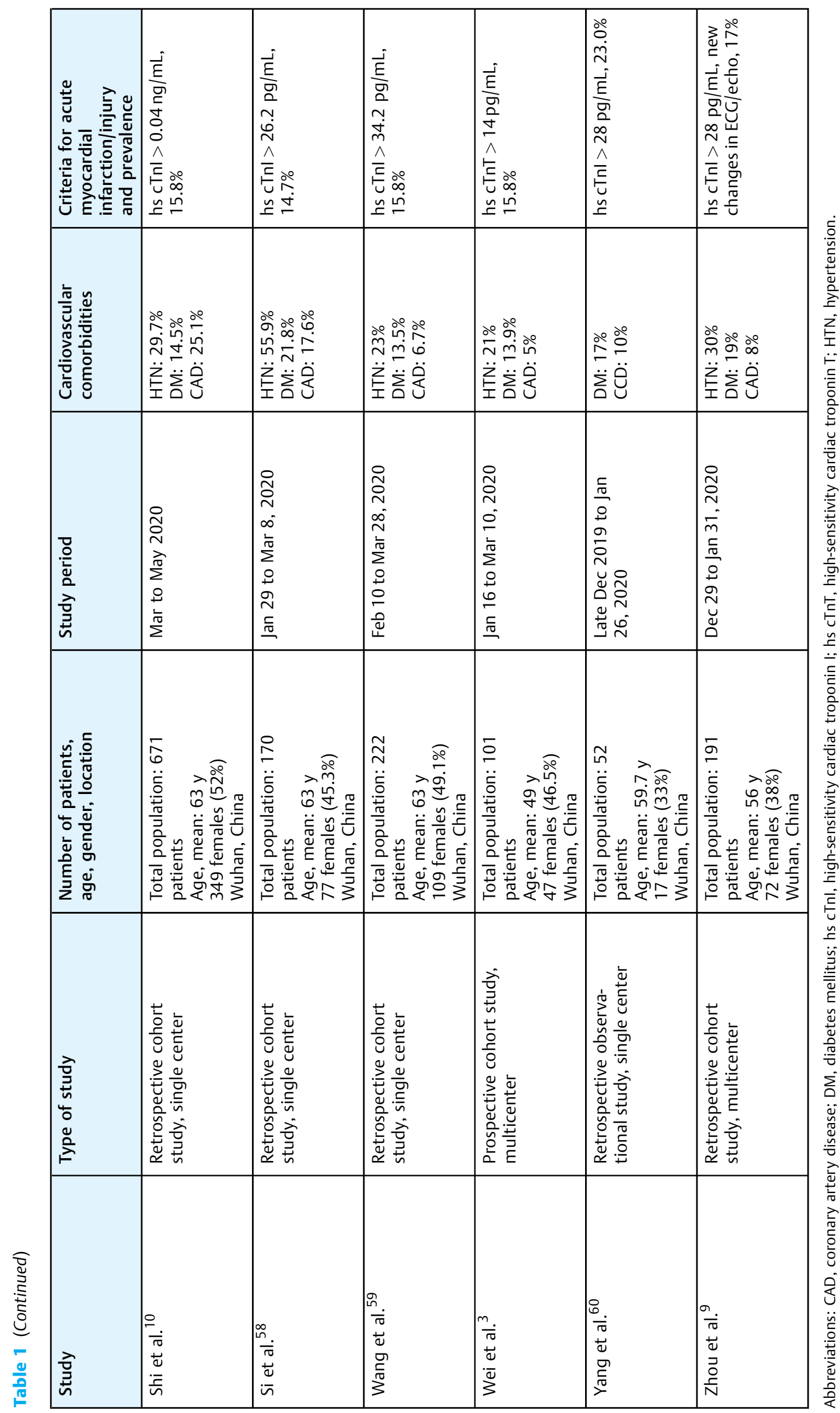


acute myocardial infarction, Lopes et al. required, in addition to elevation of cardiac biomarkers, consistent clinical presentation as well as electrocardiographic or imaging test evidence. ${ }^{8}$ Specific cutoff criteria for cardiac biomarkers are also listed in -Table 1.

In 19 studies including a total of 14,745 patients, the pooled overall prevalence of acute myocardial injury among hospitalized COVID-19 patients was 26.8\% (3,947 patients). According to Lombardi et al., predictors of cardiac injury are age and comorbidities such as hypertension, heart failure, and coronary artery disease. ${ }^{5}$ The prevalence of acute myocardial injury ranged from 6.5 to $51 \%$ in the selected studies. Univariable analysis by Zhou et al. revealed a significant association between high-sensitivity cardiac troponin I and intrahospital mortality. Additional noteworthy findings in this study were the clearly elevated levels of coagulopathy or systemic inflammation-associated markers, such as D-dimer, interleukin (IL)-6, and procalcitonin, in nonsurvivors. ${ }^{9}$ Furthermore, a multivariable analysis of 671 patients by Shi et al. confirmed an independent association of myocardial injury with an increased risk for mortality in patients with COVID-19. ${ }^{10}$ In another retrospective cohort study, both high-sensitivity cardiac troponin I (hazards ratio: 7.12, $p<0.001$ ) and CK-MB (hazards ratio: $4.86, p<0.001$ ) were found independently associated with increased 28-day allcause mortality. ${ }^{11}$

-Fig. 1 illustrates the significantly increased risk of death in patients with elevated cardiac injury markers and COVID19 in selected studies. Karbalai Saleh et al. reported that in patients with cardiac injury, elevated troponin coincided with higher levels of inflammatory makers like white blood cell count or C-reactive protein levels when compared with COVID-19 patients without cardiac injury. ${ }^{12} \mathrm{Li}$ et al. found a close correlation of immune dysregulation and myocardial injury in 182 COVID-19 patients. ${ }^{13}$ White blood cell count, neutrophil percentage, $\mathrm{CD} 3+\mathrm{T}$ cell counts, $\mathrm{CD} 4+\mathrm{T}$ cell counts, CD8 + T cell counts, NK cell counts, and procalcitonin were independently associated with myocardial injury and showed a predictive ability in the multivariate logistic regression when compared with cases without myocardial injury.

\section{Discussion}

Myocardial injury is prevalent in COVID-19-positive patients and is predictive of adverse outcomes. ${ }^{3}$ Metkus et al. reported a greater than twofold risk of mortality in critical ill patients with myocardial injury. ${ }^{14}$ Previous reports also identified higher levels of the myocardial distress markers such as troponin I or N-terminal pro-B-type natriuretic peptide (NT-proBNP) to be highly predictive for respiratory failure. ${ }^{8}$ By using nationwide registers in Denmark, Modin et al. provided evidence that COVID-19 may be associated with an increased risk of ischemic cardiovascular events. ${ }^{15}$ The incidence of acute myocardial infarction 14 days after a positive test for COVID-19 was approximately five times higher when compared with the 180 days prior to the COVID-19 diagnosis.

The exact pathophysiology of myocardial injury induced by SARS-CoV-2 is not clearly understood, but seems to be associated with severe inflammatory response, coronary microvascular ischemia, direct injury of the cardiomyocytes, and cytokine-mediated plaque destabilization. ${ }^{16,17}$ Furthermore, hypoxemia caused by respiratory failure and hemodynamic instability may favor particularly type 2 myocardial infarction caused by a mismatch of oxygen supply and demand. To meet the universal definition of myocardial infarction type 2, evidence of ischemic myocardial infarction, clinical presentation, and ECG and imaging are mandatory. Nonischemic myocardial injury may be more common in

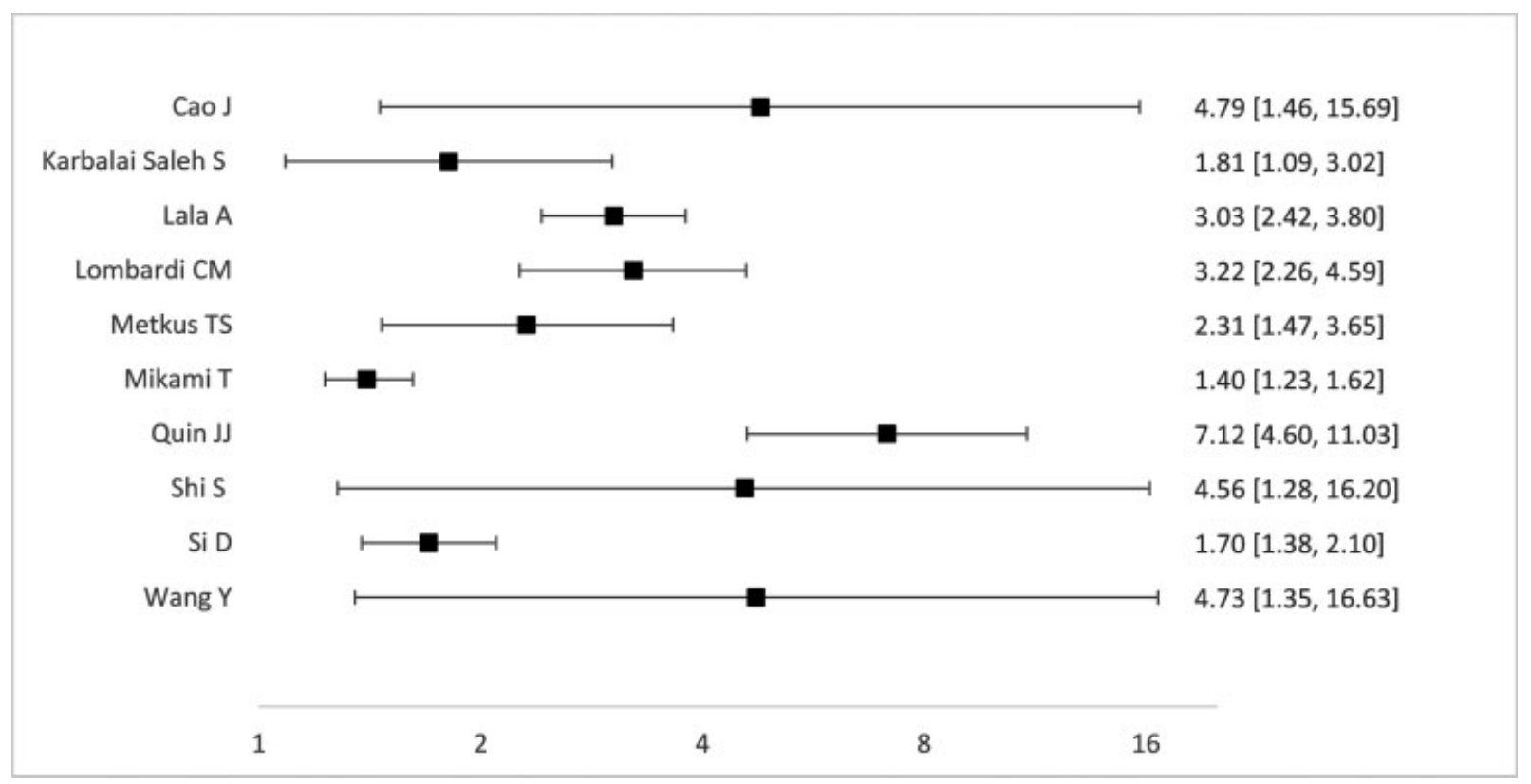

Fig. 1 Forest plot of risk of mortality for patients with cardiac injury and COVID-19. 
COVID-19. In addition to cardiac causes of nonischemic myocardial injury such as myocarditis or stress cardiomyopathy, the focus in COVID-19 may be primarily on noncardiac entities such as critical illness, severe sepsis, or pulmonary hypertension due to acute pulmonary embolism. Increased levels of troponin I and NT pro-BNP are indicative for acute myocardial stress related to the elevation of the pulmonary artery pressure and right ventricular dysfunction in patients with severe COVID-19 presentations. ${ }^{18}$

Elevations in cardiac troponin are also present in septic patients and correlate with the occurrence of impaired left ventricular function. ${ }^{19,20}$ Potential mechanisms are summarized in -Fig. 2 .

\section{Potential Mechanisms of COVID-19-Associated Myocardial Injury}

Numerous potential pathophysiological mechanisms of troponin elevation in COVID-19 patients are conceivable. Severe systemic inflammation and hypercoagulopathy are key factors in the pathophysiology of SARS-CoV-2 infection and thus another trigger of cardiovascular events. Additionally, abnormal endothelial function may increase the risk of atherosclerotic plaque disruption and myocardial infarction. ${ }^{21}$ In detail, high levels of proinflammatory cytokines, such as IL-6, IL-1, or tumor necrosis factor- $\alpha$ (TNF- $\alpha$ ), induce macrophage infiltration in the vascular wall and alter the thrombotic/fibrinolytic balance that promote the tendency for clotting. IL- 1 and TNF- $\alpha$ also suppress intrinsic anticoagulant pathways. ${ }^{22}$

A cytokine storm profile can be found in some critical ill adults, characterized by severe systemic elevation of proinflammatory cytokines and chemokines. ${ }^{23}$ The cytokine storm originates from activated TH1 cells, followed by macrophages and neutrophils infiltration, which are activated by SARS-CoV-2 after entering the respiratory epithelium. Hirano and Murakami et al. found the angiotensin 2 pathway as a potential mechanism for the uncontrolled inflammatory response and the cytokine storm. ${ }^{24}$ These proinflammatory mediators and cytokines also affect the endothelium and vascular homeostasis.

Tissue factor, expressed by stimulated endothelial cells, activates the coagulation system by the initiation of thrombin formation via activation of factor VII and $\mathrm{X} .{ }^{21}$ The endothelial cells further release von Willebrand factor causing platelet aggregation. Together with a reduced release of prostacyclin and an increased production of plasminogen activator inhibitor-1 (PAI-1), blood clotting and thrombus accumulation are promoted. ${ }^{21}$ The thrombogenicity and hemostatic imbalance related to SARS-CoV-2 infection are associated with a higher prevalence of thromboembolic cardiovascular complications. $^{25-27}$ Particularly, altered platelet function and vascular inflammation contribute to thrombotic complications. Zaid et al. provided evidence that hyperactivated platelets in COVID-19 patients favor a procoagulatory state due to inflammatory mediator release. ${ }^{28}$ Interaction of platelet receptor GPIIb/IIIa with corresponding neutrophil receptors induces the release of neutrophil extracellular traps (NETs). NETs are found in microvascular thrombi and play a role in vessel occlusion and tissue damage in COVID-19. ${ }^{29,30}$ Increased production of prothrombotic platelet-activating antibodies in response to SARS-CoV-2 was described by Althaus et al. in severe COVID-19 infection. ${ }^{31}$

Another potential mechanism is the direct myocardial damage by SARS-CoV-2, since it uses the human angiotensinconverting enzyme 2 (ACE-2) receptor for cellular entry mediated by its surface spike proteins. Taking etymological

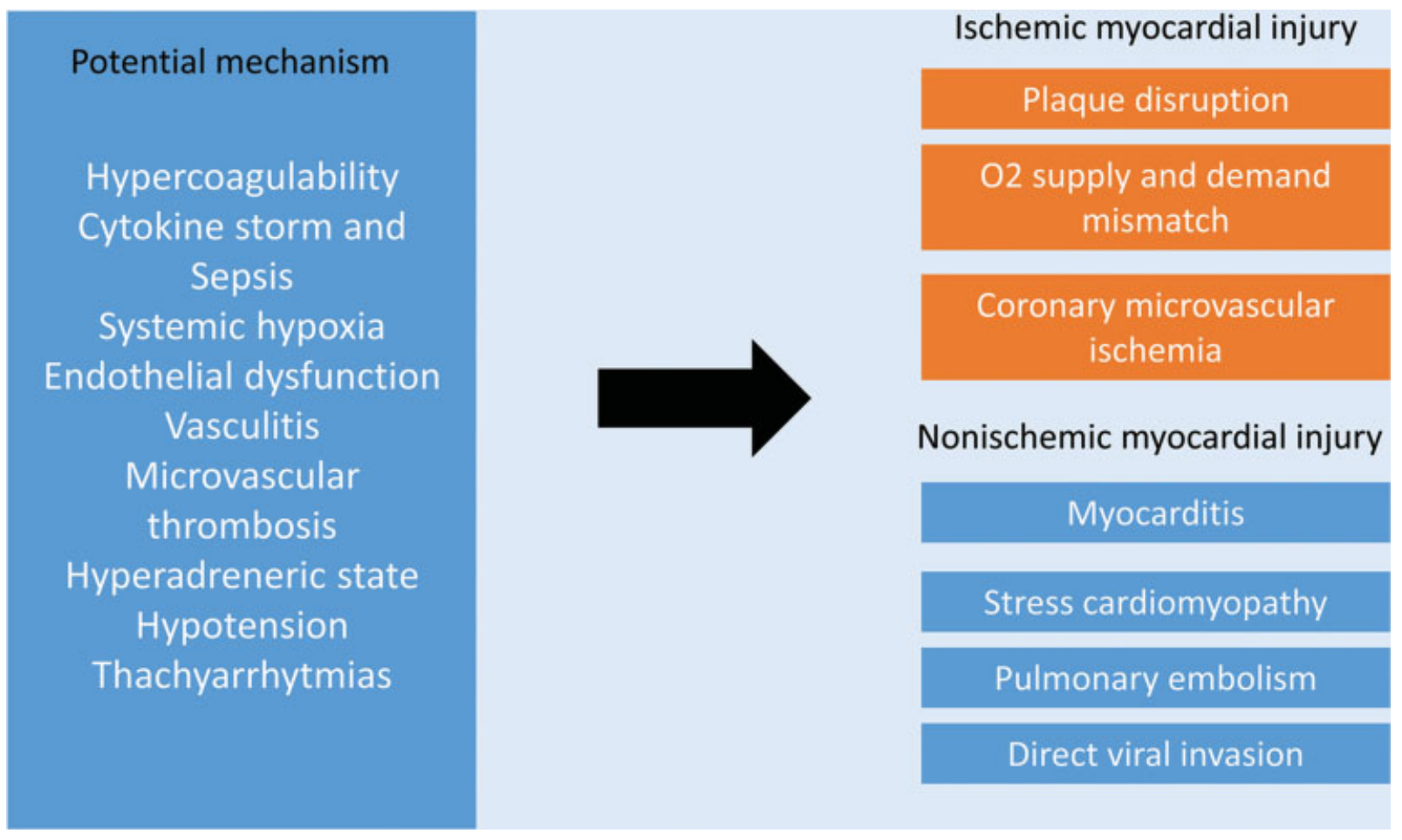

Fig. 2 Potential mechanisms of increased cardiac troponin levels above the 99th percentile upper reference limit (URL) in COVID-19. 
aspects into account, corona in Latin means "garland" or "crown" referring to the presence of its spike-like capsid under the electron microscope. ${ }^{32}$ ACE-2, a metallopeptidase, plays a crucial role in cardiovascular homeostasis and is expressed on the surface of multiple tissues like the respiratory epithelial in the lung, in cardiomyocytes and pericytes of human hearts, the kidney, the brain, and especially in vascular endothelial cells. ${ }^{33,34}$ The cardioprotective effects of ACE-2 were first described by Crackower et al., as they observed a severe left ventricular dysfunction in ACE-2 knockout mice. ${ }^{35}$ The loss of ACE-2 also raised the expression levels of hypoxia-regulated genes, indicating a myocardial stunning due to chronic hypoxia similar to coronary artery disease. SARS-CoV-2 appears to downregulate the ACE-2 by fusing with the membrane receptor on the entry and removing it from the surface. ${ }^{36}$ Thus, downregulation of ACE-2 by SARS-CoV-2 may be linked with cardiac dysfunction and heart failure. In addition, ACE inhibitors appear to increase cardiac ACE-2 gene expression and could theoretically contribute to an increased vulnerability to infection. ${ }^{37}$ However, large cohort studies by Li et al. and Mancia et al. could not confirm an association between the use of ACE inhibitors or angiotensin-receptor blockers with the risk of infection and the severity of COVID-19. ${ }^{38,39}$ In animal models, ACE-2dependent myocardial infection occurred after pulmonary infection with other coronaviruses, raising the possibility of direct damage of myocardium and vascular endothelium by the virus. ${ }^{16}$ During the Toronto SARS outbreak, Oudit et al. detected viral RNA in 35\% of autopsied heart samples. Immunohistochemical analysis further revealed increased myocardial macrophage infiltration in SARS-positive hearts. In contrast, autopsy studies found cardiac cell necrosis, but lymphocytic myocarditis could not be detected in postmortem heart samples of COVID-19 patients. ${ }^{40}$ However, magnetic resonance imaging (MRI) reports confirmed diffuse subepicardial contrast enhancement associated with myocardial edema in many patients with COVID-19. ${ }^{41}$ The cardiac MRI is an additional noninvasive diagnostic tool for the detection of possible cardiac involvement and may be useful for risk stratification and prognostication. In patients who have recovered from COVID-19, especially myocardial edema and fibrosis were found. ${ }^{42}$ This remodeling process may lead to reduced contractility in the long term.

Despite these interactions, there are also noncardiac causes of myocardial injury including pulmonary embolism and underlying critical illness, sepsis, and multisystem organ dysfunction, which could be responsible for the majority of cardiac damage.

During the COVID-19 pandemic, several studies reported a decrease in the incidence of hospitalization for acute myocardial infarction worldwide. ${ }^{43-49}$ Among patients with non-ST segment elevation myocardial infarction (NSTEMI) and STEMI, Solomon et al. noticed a decline by up to $48 \%$ in the weekly rates in March and April 2020 in northern California compared with the same week in $2019 .{ }^{43}$ In an Italian nationwide observational survey, De Rosa et al. found a reduction of 48.4\% in hospital admissions for AMI throughout March 12 to 19,2020 , when compared with the same period in 2019 , respectively, for non-STEMI of $65.1 \%$ reduction and for STEMI of $26.5 \%{ }^{46}$ Another retrospective analysis of 15 hospitals in northern Italy by De Filippo et al. observed a significant reduction in hospital admissions for ACS per day during COVID-19 pandemic compared with 2019 (18.9 vs. 13.3 daily admissions, $p<0.001) .{ }^{44}$ The ongoing French Cohort of Myocardial Infarction Evaluation (FRENCHIE) registry, in which 21 centers participated, was recently analyzed by Mesnier et al. According to their findings, admissions for acute myocardial infarction decreased by $30 \%$ from 686 to 481 between 4 weeks prior to and 4 weeks after the lockdown. ${ }^{48}$ The reduction was significant for both, STEMI (24\%, incidence rate ratio: 0.72 [95\% CI: 0.62-0.85]) and non-STEMI (35\%; 0.64 [0.55-0.76]). In Germany, the incidence and fatality rates from COVID-19 were low in March to June 2020 and a total of 207 admissions for myocardial infarction were registered in Berlin during the weeks 11 to 14 , with a $19.5 \%$ reduction (95\% CI: $3.0-33.0$ ) compared with the corresponding period in 2019 $(p=0.021){ }^{49}$ The reduction of hospitalizations for STEMI was 9 and 28\% for non-STEMI. Based on similar results in the reduction of AMI admissions in several countries, the severity of the COVID-19 pandemic can at best have a minor impact. The reasons behind the overall decrease are various and system- and patient-related factors may be partially responsible. One of the main reasons could be the fear of potential infection with the SARS-CoV-2 at the hospital. Also, the government advised the population to stay at home and minimize contact with others to a minimum. A considerable number of patients may stay in self-isolation and not attend healthcare services unless in a life-threatening emergency. Additionally, changes in physical activity may be associated with angina pectoris burden during quarantine periods. Recently, a single-center observational study by Fardman et al. indicated a post-COVID-19 rebound effect. ${ }^{51}$ In this study, a noticeable increase in hospitalizations for acute myocardial infarction after the first wave of the COVID-19 pandemic in April and May 2020 was observed.

\section{Conclusion}

Cardiac complications are common in COVID-19 and associated with adverse prognosis. Systemic inflammation and prothrombotic activity are related to myocardial injury, particularly acute myocardial infarction type 2 and nonischemic myocardial infarction. Due to its prognostic value, troponin should be assessed at the time of hospital admission in COVID-19 patients for risk stratification.

Conflict of Interests

The authors declare that they have no conflict of interest.

\section{References}

1 Gupta A, Madhavan MV, Sehgal K, et al. Extrapulmonary manifestations of COVID-19. Nat Med 2020;26(07):1017-1032

2 Chen T, Wu D, Chen H, et al. Clinical characteristics of 113 deceased patients with coronavirus disease 2019: retrospective study. BMJ 2020;368:m1091 
3 Wei JF, Huang FY, Xiong TY, et al. Acute myocardial injury is common in patients with COVID-19 and impairs their prognosis. Heart 2020;106(15):1154-1159

4 Majure DT, Gruberg L, Saba SG, Kvasnovsky C, Hirsch JS, Jauhar RNorthwell Health COVID-19 Research Consortium. Usefulness of elevated troponin to predict death in patients with COVID-19 and myocardial injury. Am J Cardiol 2021;138:100-106

5 Lombardi CM, Carubelli V, Iorio A, et al. Association of troponin levels with mortality in Italian patients hospitalized with coronavirus disease 2019: results of a multicenter study. JAMA Cardiol 2020;5(11):1274-1280

6 Li B, Yang J, Zhao F, et al. Prevalence and impact of cardiovascular metabolic diseases on COVID-19 in China. Clin Res Cardiol 2020; 109(05):531-538

7 Thygesen K, Alpert JS, Jaffe AS, et al. Executive Group on behalf of the Joint European Society of Cardiology (ESC)/American College of Cardiology (ACC)/American Heart Association (AHA)/World Heart Federation (WHF) Task Force for the Universal Definition of Myocardial Infarction. Fourth universal definition of myocardial infarction (2018). J Am Coll Cardiol 2018;72(18):2231-2264

8 Lopes RD, Macedo AVS, de Barros E Silva PGM, et al. BRACE CORONA Investigators. Effect of discontinuing vs continuing angiotensin-converting enzyme inhibitors and angiotensin ii receptor blockers on days alive and out of the hospital in patients admitted with COVID-19: a randomized clinical trial. JAMA 2021; 325(03):254-264

9 Zhou F, Yu T, Du R, et al. Clinical course and risk factors for mortality of adult inpatients with COVID-19 in Wuhan, China: a retrospective cohort study. Lancet 2020;395(10229):1054-1062

10 Shi S, Qin M, Cai Y, et al. Characteristics and clinical significance of myocardial injury in patients with severe coronavirus disease 2019. Eur Heart J 2020;41(22):2070-2079

11 Qin JJ, Cheng X, Zhou F, et al. Redefining cardiac biomarkers in predicting mortality of inpatients with COVID-19. Hypertension 2020;76(04):1104-1112

12 Karbalai Saleh S, Oraii A, Soleimani A, et al. The association between cardiac injury and outcomes in hospitalized patients with COVID-19. Intern Emerg Med 2020;15(08):1415-1424

13 Li D, Chen Y, Jia Y, et al. SARS-CoV-2-induced immune dysregulation and myocardial injury risk in China: insights from the ERSCOVID-19 study. Circ Res 2020;127(03):397-399

14 Metkus TS, Sokoll LJ, Barth AS, et al. Myocardial injury in severe COVID-19 compared with non-COVID-19 acute respiratory distress syndrome. Circulation 2021;143(06):553-565

15 Modin D, Claggett B, Sindet-Pedersen C, et al. Acute COVID-19 and the incidence of ischemic stroke and acute myocardial infarction. Circulation 2020;142(21):2080-2082

16 Oudit GY, Kassiri Z, Jiang C, et al. SARS-coronavirus modulation of myocardial ACE2 expression and inflammation in patients with SARS. Eur J Clin Invest 2009;39(07):618-625

17 Babapoor-Farrokhran S, Gill D, Walker J, Rasekhi RT, Bozorgnia B, Amanullah A. Myocardial injury and COVID-19: possible mechanisms. Life Sci 2020;253:117723

18 Rath D, Petersen-Uribe Á, Avdiu A, et al. Impaired cardiac function is associated with mortality in patients with acute COVID-19 infection. Clin Res Cardiol 2020;109(12):1491-1499

19 Mehta NJ, Khan IA, Gupta V, Jani K, Gowda RM, Smith PR. Cardiac troponin I predicts myocardial dysfunction and adverse outcome in septic shock. Int J Cardiol 2004;95(01):13-17

20 Agewall S, Giannitsis E, Jernberg T, Katus H. Troponin elevation in coronary vs. non-coronary disease. Eur Heart J 2011;32(04): 404-411

21 Libby P, Lüscher T. COVID-19 is, in the end, an endothelial disease. Eur Heart J 2020;41(32):3038-3044

22 Levi M, Thachil J, Iba T, Levy JH. Coagulation abnormalities and thrombosis in patients with COVID-19. Lancet Haematol 2020;7 (06):e438-e440
23 Hu B, Huang S, Yin L. The cytokine storm and COVID-19. J Med Virol 2021;93(01):250-256

24 Hirano T, Murakami M. COVID-19: a new virus, but a familiar receptor and cytokine release syndrome. Immunity 2020;52(05): 731-733

25 Di Minno A, Ambrosino P, Calcaterra I, Di Minno MND. COVID-19 and venous thromboembolism: a meta-analysis of literature studies. Semin Thromb Hemost 2020;46(07):763-771

26 Nannoni S, de Groot R, Bell S, Markus HS. Stroke in COVID-19: a systematic review and meta-analysis. Int J Stroke 2021;16(02): 137-149

27 Mehta JL, Calcaterra G, Bassareo PP. COVID-19, thromboembolic risk, and Virchow's triad: lesson from the past. Clin Cardiol 2020; 43(12):1362-1367

28 Zaid Y, Puhm F, Allaeys I, et al. Platelets can associate with SARSCoV-2 RNA and are hyperactivated in COVID-19. Circ Res 2020; 127(11):1404-1418

29 Nicolai L, Leunig A, Brambs S, et al. Immunothrombotic dysregulation in COVID-19 pneumonia is associated with respiratory failure and coagulopathy. Circulation 2020;142(12): 1176-1189

30 Gaertner F, Ahmad Z, Rosenberger G, et al. Migrating platelets are mechano-scavengers that collect and bundle bacteria. Cell 2017; 171(06):1368-1382.e23

31 Althaus K, Marini I, Zlamal J, et al. Antibody-induced procoagulant platelets in severe COVID-19 infection. Blood 2021;137(08): 1061-1071

32 Zhang SF, Tuo JL, Huang XB, et al. Epidemiology characteristics of human coronaviruses in patients with respiratory infection symptoms and phylogenetic analysis of HCoV-OC43 during 2010-2015 in Guangzhou. PLoS One 2018;13(01):e0191789

33 Chen L, Li X, Chen M, Feng Y, Xiong C. The ACE2 expression in human heart indicates new potential mechanism of heart injury among patients infected with SARS-CoV-2. Cardiovasc Res 2020; 116(06):1097-1100

34 Gheblawi M, Wang K, Viveiros A, et al. Angiotensin-converting enzyme 2: SARS-CoV-2 receptor and regulator of the reninangiotensin system: celebrating the 20th anniversary of the discovery of ACE2. Circ Res 2020;126(10):1456-1474

35 Crackower MA, Sarao R, Oudit GY, et al. Angiotensin-converting enzyme 2 is an essential regulator of heart function. Nature 2002; 417(6891):822-828

36 Zhang H, Penninger JM, Li Y, Zhong N, Slutsky AS. Angiotensinconverting enzyme 2 (ACE2) as a SARS-CoV-2 receptor: molecular mechanisms and potential therapeutic target. Intensive Care Med 2020;46(04):586-590

37 Ferrario CM, Jessup J, Chappell MC, et al. Effect of angiotensinconverting enzyme inhibition and angiotensin II receptor blockers on cardiac angiotensin-converting enzyme 2 . Circulation 2005;111(20):2605-2610

38 Li J, Wang X, Chen J, Zhang H, Deng A. Association of reninangiotensin system inhibitors with severity or risk of death in patients with hypertension hospitalized for coronavirus disease 2019 (COVID-19) infection in Wuhan, China. JAMA Cardiol 2020;5 (07):825-830

39 Mancia G, Rea F, Ludergnani M, Apolone G, Corrao G. Reninangiotensin-aldosterone system blockers and the risk of COVID19. N Engl J Med 2020;382(25):2431-2440

40 Fox SE, Akmatbekov A, Harbert JL, Li G, Quincy Brown J, Vander Heide RS. Pulmonary and cardiac pathology in African American patients with COVID-19: an autopsy series from New Orleans. Lancet Respir Med 2020;8(07):681-686

41 Knight DS, Kotecha T, Razvi Y, et al. COVID-19: myocardial injury in survivors. Circulation 2020;142(11):1120-1122

42 Huang L, Zhao P, Tang D, et al. Cardiac involvement in patients recovered from COVID-2019 identified using magnetic resonance imaging. JACC Cardiovasc Imaging 2020;13(11):2330-2339 
43 Solomon MD, McNulty EJ, Rana JS, et al. The COVID-19 pandemic and the incidence of acute myocardial infarction. $\mathrm{N}$ Engl J Med 2020;383(07):691-693

44 De Filippo O, D'Ascenzo F, Angelini F, et al. Reduced rate of hospital admissions for ACS during COVID-19 outbreak in northern Italy. $\mathrm{N}$ Engl J Med 2020;383(01):88-89

45 Meenakshisundaram R, Senthilkumaran S, Thirumalaikolundusubramanian $\mathrm{P}$, et al. Status of acute myocardial infarction in southern India during COVID-19 lockdown: a multicentric study. Mayo Clin Proc Innov Qual Outcomes 2020;4(05):506-510

46 De Rosa S, Spaccarotella C, Basso C, et al. Società Italiana di Cardiologia and the CCU Academy investigators group. Reduction of hospitalizations for myocardial infarction in Italy in the COVID19 era. Eur Heart J 2020;41(22):2083-2088

47 Metzler B, Siostrzonek P, Binder RK, Bauer A, Reinstadler SJ. Decline of acute coronary syndrome admissions in Austria since the outbreak of COVID-19: the pandemic response causes cardiac collateral damage. Eur Heart J 2020;41(19):1852-1853

48 Mesnier J, Cottin Y, Coste P, et al. Hospital admissions for acute myocardial infarction before and after lockdown according to regional prevalence of COVID-19 and patient profile in France: a registry study. Lancet Public Health 2020;5(10):e536-e542

49 Dreger $\mathrm{H}$, Bruch L, Maier B, Schühlen H. Acute myocardial infarction admissions in berlin during the COVID-19 pandemic. Dtsch Arztebl Int 2020;117(35-36):597-598

50 Cao J, Zheng Y, Luo Z, et al. Myocardial injury and COVID-19: Serum hs-cTnI level in risk stratification and the prediction of 30 day fatality in COVID-19 patients with no prior cardiovascular disease. Theranostics 2020;10(21):9663-9673

51 Fardman A, Oren D, Berkovitch A, et al. Post COVID-19 Acute Myocardial Infarction Rebound. Can J Cardiol 2020;36(11):1832. e15-1832.e16
52 Cecconi M, Piovani D, Brunetta E, et al. Early Predictors of Clinical Deterioration in a Cohort of 239 Patients Hospitalized for Covid-19 Infection in Lombardy, Italy. J Clin Med 2020;9 (05)

53 Cummings MJ, Baldwin MR, Abrams D, et al. Epidemiology, clinical course, and outcomes of critically ill adults with COVID-19 in New York City: a prospective cohort study. Lancet 2020;395(10239): 1763-1770

54 Guo T, Fan Y, Chen M, et al. Cardiovascular implications of fatal outcomes of patients with coronavirus disease 2019 (COVID-19). JAMA Cardiol 2020;5(07):811-818

55 Huang C, Wang Y, Li X, et al. Clinical features of patients infected with 2019 novel coronavirus in Wuhan, China. Lancet 2020;395 (10223):497-506

56 Lala A, Johnson KW, Januzzi JL, et al. Mount Sinai COVID Informatics Center. Prevalence and impact of myocardial injury in patients hospitalized with COVID-19 infection. J Am Coll Cardiol 2020;76(05):533-546

57 Ni W, Yang X, Liu J, et al. Acute myocardial injury at hospital admission is associated with all-cause mortality in COVID-19. J Am Coll Cardiol 2020;76(01):124-125

58 Si D, Du B, Ni L, et al. Death, discharge and arrhythmias among patients with COVID-19 and cardiac injury. CMAJ 2020;192(28): E791-E798

59 Wang Y, Zheng Y, Tong Q et al. Cardiac injury and clinical course of patients with coronavirus disease 2019. Front Cardiovasc Med 2020;7:147

60 Yang X, Yu Y, Xu J, et al. Clinical course and outcomes of critically ill patients with SARS-CoV-2 pneumonia in Wuhan, China: a single-centered, retrospective, observational study. Lancet Respir Med 2020;8(05):475-481 